\title{
The Effect of 12-Week Passive Aquatic Bodywork on Sympathovagal Balance of Obese Youth
}

\author{
Ertan Tufekcioglu ${ }^{1}$, Mustafa Sait Erzeybek ${ }^{2}$, Fatih Kaya ${ }^{3}$, Goksel Ozan $^{4}$ \\ ${ }^{1}$ Physical Education Department, King Fahd University of Petroleum \& Minerals, Saudi Arabia \\ ${ }^{2}$ Department of Physical Education and Sports, Dumlupinar University, Turkey \\ ${ }^{3}$ Department of Physical Education and Sports, Faculty of Education, Erzincan University, Turkey \\ ${ }^{4}$ ASFA Foundation for Education, Istanbul, Turkey
}

Correspondence: Mustafa Sait Erzeybek, Department of Physical Education and Sports, Dumlupinar University, Turkey.

Received: December 28, 2017

Accepted: January 18, $2018 \quad$ Online Published: January 29, 2018

doi:10.11114/jets.v6i2.2963

URL: https://doi.org/10.11114/jets.v6i2.2963

\begin{abstract}
Background: Obesity has been identified as a global epidemic that is associated with numerous comorbidities such as type 2 diabetes, hypertension, cancer, cardiovascular disease. We have investigated the effects of Watsu ${ }^{\circledR}$ therapy and Immersion on HRV parameters of obese male subjects.

Methods and Results: Volunteer 34 obese subjects with BMI above 30 without any other chronic health issues were included (age, 18.30 \pm .31 , height, 166.02 \pm 29.60 , BMI, 36.54 \pm 5.96 ). Randomized controlled trial was conducted with Watsu and Immersion protocols whereas control group received no therapy in water or on land for 12 weeks. A baseline HRV was recorded 5 minutes in four different circumstances. Polar H7 heart rate sensor and digital standardized HRV signal processing software were used to record the R-R intervals in millisecond. The repeated measures were used to compare the conditions between the therapies. The HRV analyses were performed using three-way Mixed ANOVA. Multiple comparisons were done with a Bonferroni adjustment of the alpha level (0.05). The collective results of HF showed significant negative effect $(13.01 \pm 1.36,9.99 \pm 1.11)$ and HF laying supine value $(20.62 \pm 2.22)$ was the highest $(\mathrm{p}<0.05)$.
\end{abstract}

Conclusion: A significant inverse correlation was found between HRV values and both therapeutic interventions. Counterintuitively, 12-week of watsu and immersion therapies decreased LF and HF.

Keywords: hearth rate variability, watsu, obesity, immersion, aquatic therapy

\section{Introduction}

Obesity has been identified as a global epidemic that is associated with numerous comorbidities such as type 2 diabetes, hypertension, cancer, cardiovascular disease (CVD) and sleep apnea (Eckel et al., 2004). The mechanisms that effect autonomic nervous system (ANS) functions of obese patients are not very clear (Hall et al 2002, Kalil \& Haynes 2012, Smit \& Minson 2012, Canale et al 2013).

ANS is a key factor in the regulation of energy balance glucose and adipose metabolisms, and body fat storage (Guariono et al 2017). In addition, obesity is considered as an independent risk factor for CVD and an important cause of ANS dysfunction (Poirier and Eckel, 2002). ANS dysfunction is considered an important mediator in the development of obesity-associated disease and insulin resistance (Hall et al. 2015).

ANS is a control system that acts largely unconsciously and regulates bodily functions such as heart rate, digestion, respiratory rate, pupillary response, urination, and sexual arousal (Kenney \& Ganta 2014). The function of ANS can be assessed via a noninvasive Hearth Rate Variability (HRV) analyses (Task Force 1996). HRV is the variation of beat-to-beat R-R intervals recorded by an ECG (electro cardio gram) (Metelka 2014) and one of the most promising, amenable quantitative markers of the autonomic balance (Kamath 2015, Buchheit and Gindre 2006, Norris 2005). HRV has become very popular, as it opens a wide range of possibilities for the easy and noninvasive investigation of autonomic function in humans (Dong 2016, Mc Craty \& Shaffer 2015, Thayer et al 2009). Alterations in HRV are predictive and associated with various cardiovascular diseases and pathological conditions, diagnosis of 
over-training, reflecting recovery status and aerobic performance (Ziegler et al 2006, Kluttig et al 2010, Adamson et al 2004, Al Haddad et al 2010). There is more correlation between body mass index (BMI) and HRV than regular physical activity (Rennie et al 2003). The controversial data on the associations between BMI and HRV could be the distribution of body fat (Windham et al. 2012). The excessive accumulation of fat tissue around the waist area leads to morphological changes in cardiac structure and function, even in the absence of other risks of heart disease (Poirier et al., 2004). HRV indices are displayed in time and frequency domain parameters (Shaffer \& Gingsberg 2017, Task Force 1996). The frequency domain analysis permits the study of the sympathetic and parasympathetic contributions of autonomic control with some degree of separation (Hadase et al 2004). The selected frequency-domain measures in normalized units (n.u.) are (1) very low frequency power (VLFMS2) from 0.00 to $0.04 \mathrm{~Hz}$; (2) low frequency power (LF) from 0.04 to $0.15 \mathrm{~Hz}$; and (3) high frequency power (HF) from 0.15 to $0.40 \mathrm{~Hz}$ (Shaffer \& Gingsberg 2017).

The findings of a number of studies (Schitter et al. 2015, Stan 2013, Kamioka et al. 2010, Becker 2009, Geytenbeek 2002) indicated that numerous psychological and physical benefits of aquatic physical therapy. Many pyschological and functional improvements can be achieved via aquatic therapies (Inoue 2013, Kesiktas et al 2004). The increased clinical implementation of watsu therapy in interdisciplinary treatment indicates a growing acceptance of this body-based complementary therapeutic intervention. Watsu ${ }^{\circledR}$ has been recognized and implemented as a relaxing therapy in many countries as a component in multimodal treatment settings focusing on posttraumatic stress disorder, and anxiety (Cole \& Becker 2011, Dull 2008). The clear challenge in aquatic bodywork field especially in innovative watsu ${ }^{\circledR}$ therapy and its ramifications is the absence of studies with standardized protocols. Positive effects of manipulation and trigger points therapies on muscle relaxation and psychological state were observed (Zimmerman 2015, Bijari et al 2012, Sherman et al 2011, Björnsdotter et al 2010, Ditzen et al 2007, Moyer et al. 2004). In the preliminary studies, the positive effect of watsu ${ }^{\circledR}$ therapy on flexibility, relaxation and ANS activity was observed (Tufekcioglu and Hassanain 2016, Barbosa et al 2014, Tufekcioglu et al 2010). Watsu has been recommended for chronic pain and fibromyalgia (Faull 2005), depression (Maczkowiak et al 2007) and for patients with hemiparesis, multiple sclerosis, cerebral palsy, and spinal cord injury (Chon et al 2009).

Immersion may be beneficial in certain populations (Ronda 2014, Gruner 2009, Cider et al 2006] due to reduced gravitational forces and hydrodynamics. Chronic pain, cardiac conditions, hypothalamic-pituitary-adrenal axis activity to alleviate stress related disorders and obesity could be regarded as potential indications for immersion (Kelly and Kirschenbau 2011, Cider et al 2006, Kjellgren et al 2001). Water immersion triggers parasympathetic activity with colder temperatures likely to be more effective (Al Haddad 2010)

However, the effect of aquatic physiotherapeutic interventions including watsu therapy on cardiac autonomic modulation of the heart in obesity have not been investigated in a large-scale randomized trial. In our preliminary study, we hypothesized that Watsu® therapy has deeper effect on HRV of obese participants, compare to warm water immersion. HRV can reflect psychomotor state determining cardiac locomotor synchronization (CLS). To date, no aquatic therapy research has been done on the techniques that have potential improving effects on CLS. Therefore, purpose of this study was to assess and compare the effect of manipulative Watsu ${ }^{\circledR}$ therapy and passive immersion protocols on HRV parameters in steady, locomotor and non locomotor recordings.

\section{Methods}

We conducted the randomized controlled trial at the KFUPM college to investigate the effects of Watsu (W) and Immersion (I) in water on HRV parameters among obese peopling. Complete written and verbal information about the study were provided and written consent was obtained prior to participation. Volunteer 34 obese persons with BMI above 30 without any other chronic health issues, habit of smoking and alcohol were included. Participants with any disease histories that are known to affect the autonomic cardiac function, such as CV (cardio vascular) dysfunction, neurological diseases, or endocrine disorders, or if they are known to be taking medications were not involved in this study. The anthropometric values were collected using InBody 370, a body composition analyzer made by Biospace Co. in South Korea.

\subsection{Experimental Design}

Randomized controlled trial was conducted with Watsu and Immersion protocols whereas control group received no therapy in water or on land for 12 weeks. Collection of Data: We applied the following same procedure one day before and after the experimental therapy period. BP (blood pressure) was measured after 10 minutes rest in a silent room, right before HRV data recordings. A baseline HRV was recorded 5 minutes in each circumstances: 1. Laying supine (horizontal). 2. Sitting (vertical). 3. Walking (locomotor). 4. Cycling (non locomotor). All participants maintained food intake routine, avoided exercise and caffeine consumption during the previous 24 hours preceding the measurements. Participants consumed $500 \mathrm{~mL}$ of water 2 hours before the measurements. Polar $\mathrm{H} 7$ heart rate sensor and digital standardized HRV signal processing software called HRV+ were used to record the R-R intervals in millisecond. iPad-2 
tablet pc that fully supports Bluetooth Smart (Bluetooth 4.0) were used for screening R-R intervals in ms. A Landice, model L-7 treadmill was used for locomotor HRV recordings at $4.5 \mathrm{~km} / \mathrm{hr}$ walking speed. A Monark, model 686 cycle ergometer was used for non-locomotor HRV recordings at $50 \mathrm{~W}$ pedaling resistance and at $55 \mathrm{rpm}$. The obtained ECG data files were then transmitted to a personal computer as txt files. The frequency domain indices of HRV (VLFMS2, LF and HF N.U.) were calculated.

The measurements were initiated after participants confirmed positive feelings of four or more points on the 5-point scale. The following steps reinforced quality control of data collection: 1. Spectra 360 Electrode Gel was used on the skin before attaching the Polar $\mathrm{H} 7$ heart rate sensor for the continuity of precise signal detection. 2. Low power filter were performed in order to eliminate the noise in the recordings and then HRV indices in frequency domain were obtained using Kubios HRV 2.2 (bio-signal analyzing software)

\subsection{Intervention Protocol}

Each watsu therapy session started with a brief verbal description of the procedure. W.A.B.A. (Worldwide Aquatic Bodywork Association) certified professional watsu practitioner applied watsu-2 therapy protocol that includes stretches, trunk rotation, joint mobilization, vertebral traction/lengthening, deep breathing and pressure point work and rocking techniques in water. Immersion group spent the same time immersed without movement and/or receiving any therapy in shoulder depth warm water. Immersion and watsu therapies were performed for 30 minutes, twice a week for 12 weeks, between 5:00-7:00 pm in an indoor swimming pool. Temperatures of the pool water and air were $32^{\circ} \mathrm{C}$ and $29^{\circ} \mathrm{C}$. Post-intervention: The aforementioned procedure for the baseline data collection was performed one day after the experimental therapy protocol.

\subsection{Statistical Analysis}

The effect of watsu therapy and immersion were analyzed separately for each measurement in four positions (laying supine, sitting, walking, cycling respectively). The pretest and posttest time period including repeated measures were used to compare the conditions between the therapies. The frequency domain parameters (VLFMS2, LF, HF N.U.) were evaluated using three-way Mixed ANOVA (one between- and two within-subjects factors). Analyses were done with checks for sphericity. Multiple comparisons were performed with a Bonferroni adjustment of the alpha level (0.05). Mean and standart error were used for all the parameters. The level of significance was determined at $\mathrm{p} \leq 0.05$ level. SPSS 20.0 computer program was used for all the statistical analyses.

\section{Results}

Table 1. Average anthropometric measurements and BMI values of the participants in each group (Mean \pm SD)

\begin{tabular}{lrll}
\hline Group & Age (year)Height $(\mathrm{cm})$ & Weight $(\mathrm{kg})$ & BMI $\left(\mathrm{w} / \mathrm{h}^{2}\right)$ \\
Watsu $(\mathrm{n}=13)$ & $18.16 \pm .27$ & $171.31 \pm 6.54$ & $108.05 \pm 20.5236 .58 \pm 5.58$ \\
Immersion $(\mathrm{n}=11)$ & $18.34 \pm .34$ & $170.27 \pm 5.39$ & $110.40 \pm 23.6138 .01 \pm 8.03$ \\
Control $(\mathrm{n}=10)$ & $18.45 \pm .26$ & $154.46 \pm 53.96102 .16 \pm 15.1734 .86 \pm 3.34$ \\
Total $(\mathrm{N}=34)$ & $18.30 \pm .31$ & $166.02 \pm 29.60107 .08 \pm 19.9036 .54 \pm 5.96$ \\
\hline
\end{tabular}

No significant differences $(\mathrm{p}>0.05)$ were seen in the baseline recordings of HF N.U. values of each groups (Watsu $=11.99 \pm 1.68$, Immersion $=10.54 \pm 1.83$, Control $=11.99 \pm 1.92$ ).

Considering the collective condition results of HF N.U. value (20.62 \pm 2.22 ), in laying supine (horizontal) are the highest $(\mathrm{p}<0.05)$ and decreased gradually in each following circumstances; sitting (vertical) $11.60 \pm 1.32$, walking (locomotor) $7.27 \pm 0.92$ and cycling (nonlocomotor) $6.52 \pm 0.66$ (Figure 1). Findings are similar ( $p>0.05$ ) to the HF N.U. value of each group (Figure 2).

The collective results of HF N.U. showed significant negative effect from pre to post test $(13.01 \pm 1.36,9.99 \pm 1.11$, $(p<0.05))$. Significant decrease were also found in all groups results $(p>0.05)$, which is contrary to our hypothesis (Figure 1).

In comparison of the groups, watsu group HF N.U. showed improvement only in the walking (locomotor) results, whereas immersion group improvement was only in cycling (nonlocomotor) results (Figure 2). However, the both group improvements are not significant ( $>0.05$ ). In addition, control group did not show any improvements in all condition results (see figure 2). 


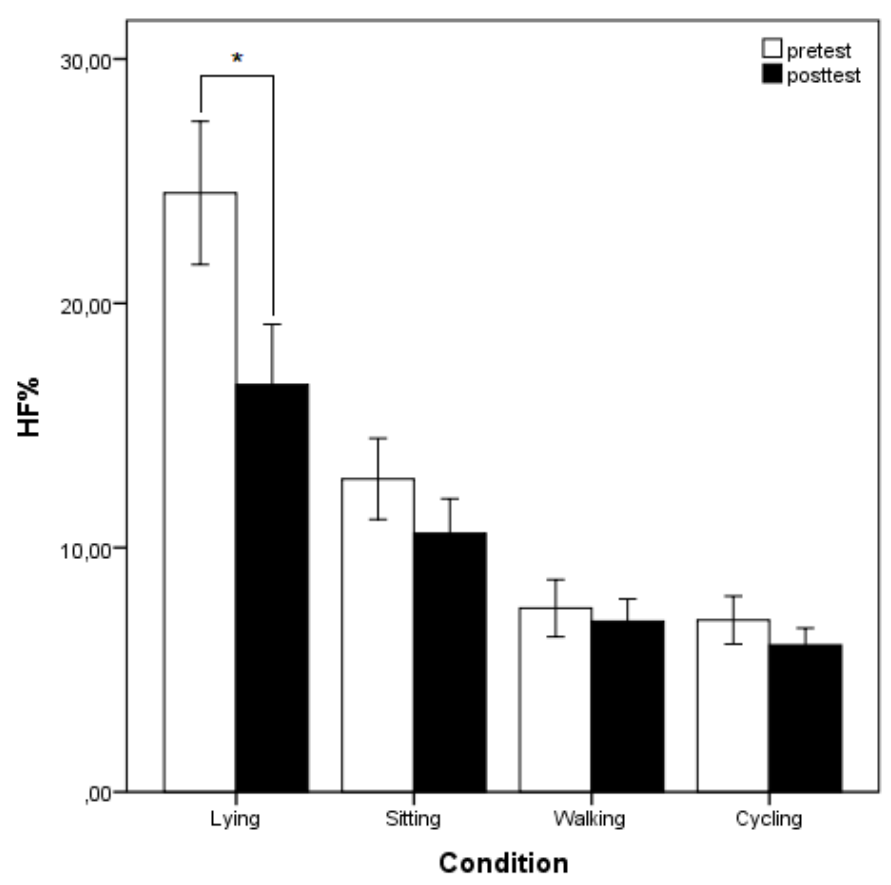

Figure 1. Collective HF N.U. recording results in all conditions (*p<.05), (+/-1 SE)

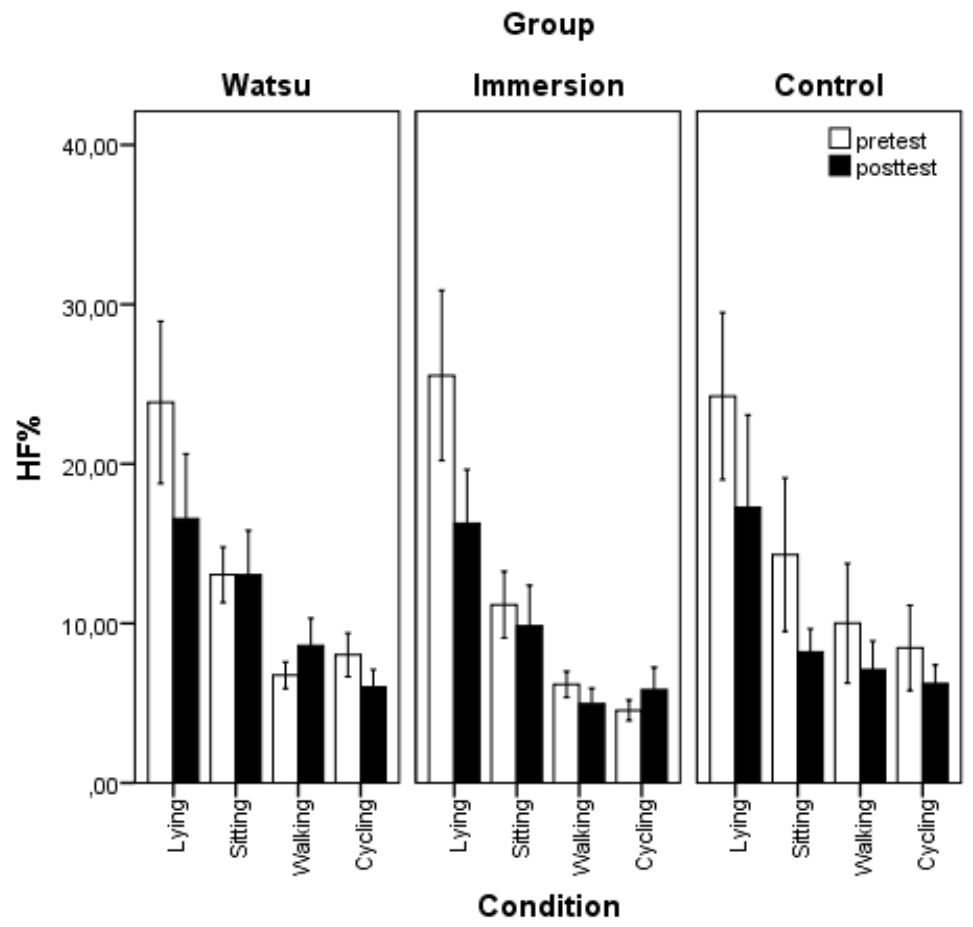

Figure 2. Groups HF N.U. average values in different recording conditions (+/-1 SE)

No significant differences were observed in LF N.U. of each group: Watsu=29.84 \pm 1.80 , Immersion $=31.63 \pm 1.96$, control=30.35 \pm 2.05 ( $>>0.05)$. The LF value showed an insignificant increase in Immersion group cycling (non locomotor) and Control group laying supine (horizontal) position ( $>0.05$ ). The pre to posttest changes are similar for the groups in each circumstances (see Figure 3) 


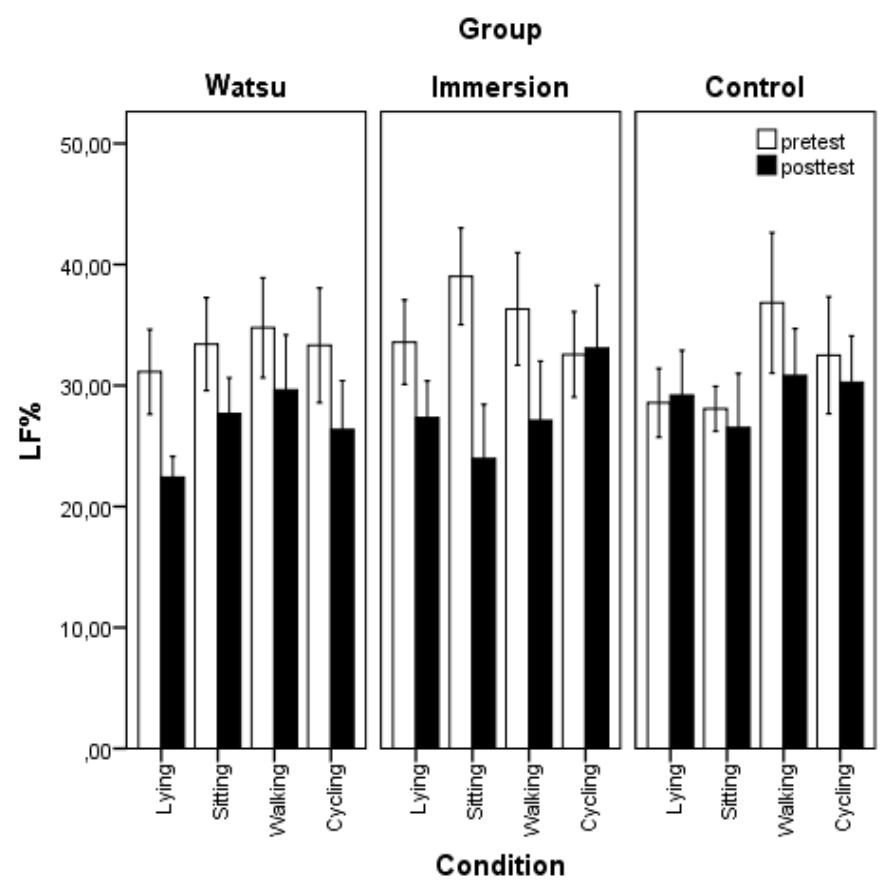

Figure 3. Groups LF N.U. average values in different recording conditions (+/-1 SE)

There was no difference between the groups $\operatorname{VLF~} \mathrm{ms}^{2}$ (nu) values: Watsu ${ }^{\circledR}=58.13 \pm 2.71$, Immersion $=57.78 \pm 2.95$, Control=57.72 \pm 3.09 ( $p>0.05$ ). Collective result of $\mathrm{VLF} \mathrm{ms}{ }^{2}$ (nu) increased from 53.65 \pm 2.06 to $62.09 \pm 1.90$ ( $p<0.05$ ). This is similar in each group ( $>>0.05)$. There was a gradual increase in order of four collective recording conditions VLF $\mathrm{ms}^{2}: 50.53 \pm 2.46,58.56 \pm 1.84,60.24 \pm 2.74,62.16 \pm 2.53$ respectively. The only significance was observed between horizontal and other recording conditions $(\mathrm{p}<0.05$, Figure 4$)$. These changes are similar in each group.

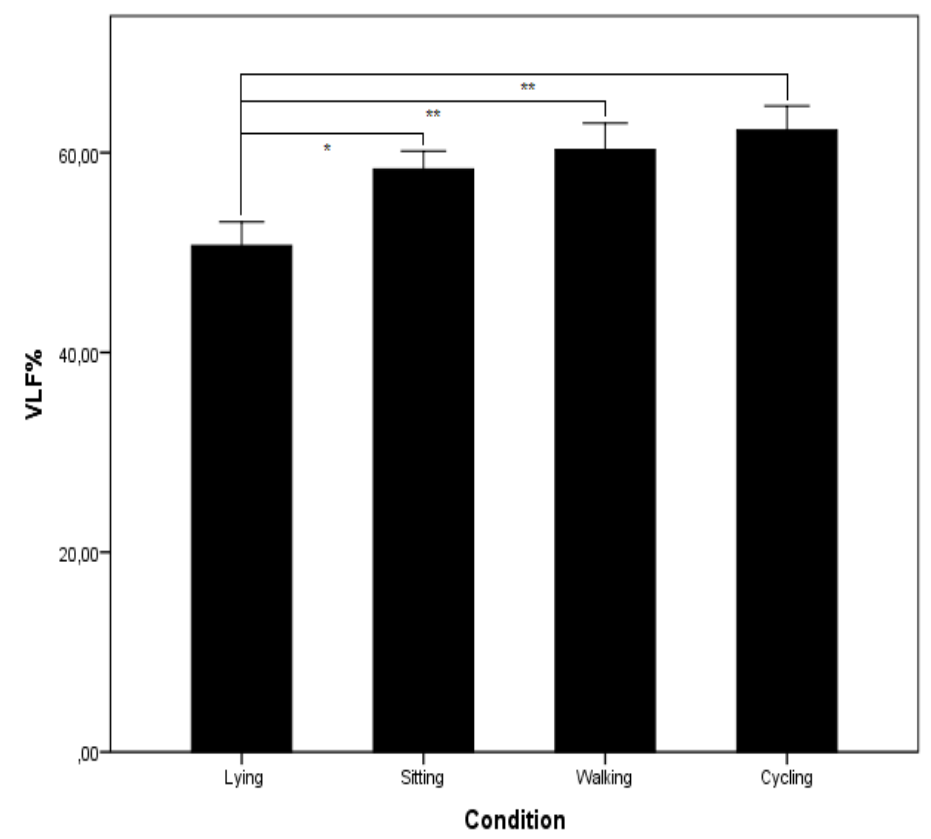

Figure 4. VLF $\mathrm{ms}^{2}$ (nu) values in all conditions $(* \mathrm{p}<.05, * * \mathrm{p}<.01),(+/-1 \mathrm{SE})$

VLF $\mathrm{ms}^{2}$ result of each groups in each conditions are similar ( $p>0.05$, Figure 5) 


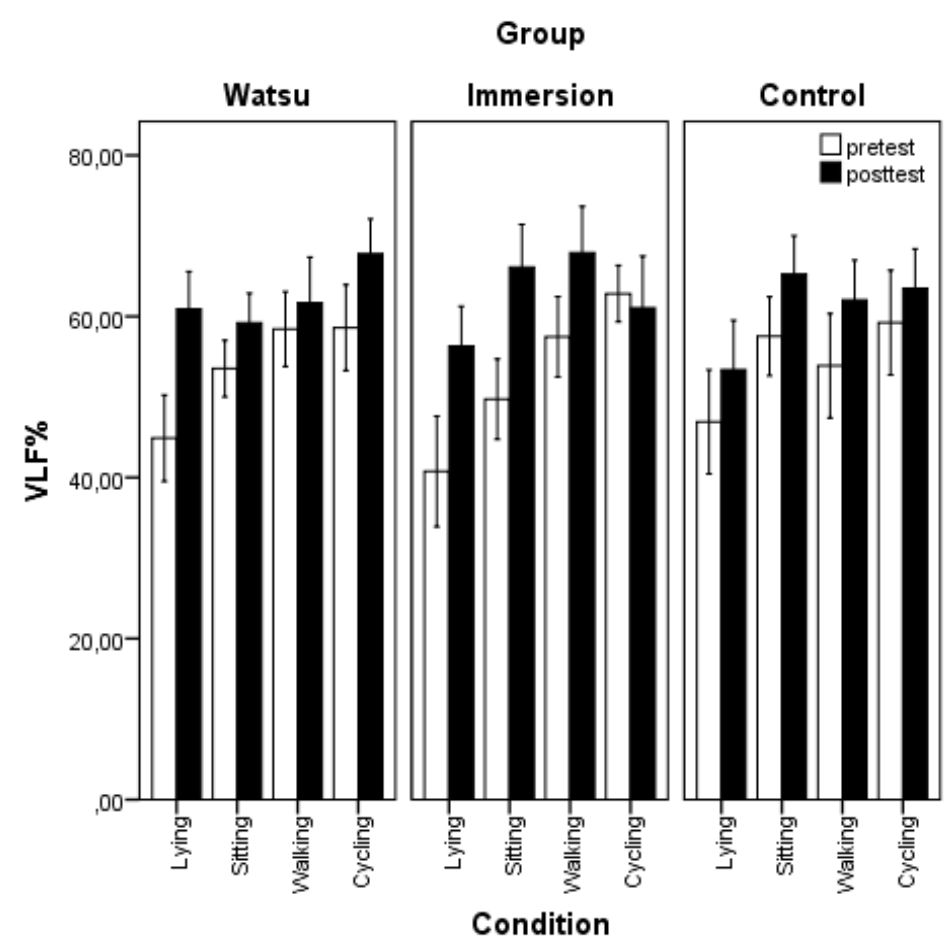

\section{Discussion}

Figure 5. VLF $\mathrm{ms}^{2}$ values in all groups (+/-1 SE)

The correlation between relaxation and enhanced HRV indices especially in healthy people were revealed (Task Force 1996). However, many in vivo studies related to HRV resulted in unstable interpretation of the link between HRV, interventions and the interplay between ANS branches of different populations. The hyperthyroid patients' revealed significant differences compared to the controls in the HRV parameters (Chen et al 2006). The adaptive resources of ANS modulation is insufficient in fibromyalgia patients and in children with CP (Zamuner et al 2015, Kerppers et al 2009). Another study reported autonomic cardiac hypo responsivity to orthostatic stress in the obese group (Perini and Veicsteinas 2003).

Improved cardio vagal baroreflex sensitivity during water immersion increases PNS tone and HRV (Laurent et al 2008). Watsu is considered to be one of the promising method for acceleration in recovery, conservative and restorative functions and the activation of afferent C-tactile fibers (Schitter et al 2015). Mooventhan and Nivethitha (2014) suggested that balanced psychomotor state with increased sympathovagal balance and level of serotonin is due to the warmth and the massage effect of water.

Water immersion to shoulder-depth was shown to be PNS activator in many studies with colder temperatures to be likely more beneficial (Ottone 2014, Nagasawa et al 2001, Soni et al 2016, Kesiktas et al. 2004). In addition, passive immersion contributes to maximize muscle relaxation that is associated with a better vagal modulation i.e. enhanced HRV indices (Cider et al 2006, Schipke 2001, Petrofsky et al 2003). Watsu components may also lead to dampened muscle tone via vagal modulation as a side effect of vestibular system activation (Cole \& Becker 2011).

The obese group presented higher BP and HR values at rest and autonomic impairment, characterized by a reduction in parasympathetic activity and relative predominance of sympathetic activity. The high value of the LFnu index and decrease in the obese group pointed to relative sympathetic predominance in these individuals (Rossi et al 2015).

The positive correlation between HF n.u. and LF n.u. shown by Goldstein et al (2011) was supported by our study. Del Paso et al. (2013) also showed LF as an indicator of PNS activity and Rossi et al. (2015) showed decreased LF value in obese group pointing sympathetic predominance which is in line with our study. We also confirm that LF component is no longer referred to a sympathetic index at all. Therefore, strong correlation found between HF n.u. and LF n.u. values in our study favors the abovementioned evidences for the current definitions of LF and VLF.

In our study, a significant inverse correlation was found between HRV values and both therapeutic interventions one of which included relaxing rotational stretches (Umphred 1995). Similar significant increase in VLF $\mathrm{ms}^{2}$ after 12-week aquatic interventions was observed in line with another study (Becker 2009) suggesting warm water immersion significantly raises VLF $\mathrm{ms}^{2}$. 
In contrast to what preliminary studies showed in this first study with obese individuals, 12-week of watsu and immersion therapies decreased LF (n.u.) and HF n.u. Becker et al (2009) showed that exposure to warm water is associated with a significant decline in HF n.u. power in healthy individuals. In addition to that, in our study walking HF n.u. after watsu and cycling HF n.u. after immersion showed insignificant increase. Our findings illuminates the possible effect of aquatic interventions on psychomotor state in relation with CLS.

Due to the negative effects seen in all groups during the study period, we cannot infer causation from our study. Control group HF and LF n.u. decreased significantly (Figure 2,3). Investigating the role that obesity plays in contributing to the etiology of ANS imbalance require also a bio-psychological perspective. These first findings of HRV on obese young individuals can encourage further studies of water therapies in different settings. Our study also favored using LF/HF ratio together with HF n.u. and LF n.u. due to the conflicting results. Researches addressing sympathovagal balance should include $\mathrm{LF} / \mathrm{HF}$ ratio due to possible distortion of the estimation of LF and HF power in absolute units (ms2). We cannot infer direct positive link between the dysregulation of the autonomic nervous system and the water therapies in this first study. A more complete understanding of the role of warm water therapies and the SNS in obesity is necessary.

However, the inverse relation between ANS balance or HRV indices and both aquatic therapies could also be attributed to external factors such as having to fit the therapy sessions into daily schedule that may have played a role in. Such changes in perception can affect the emotions therefore ANS modulation.

To know the exact time of changes in HRV may help better understand of the possible variables effecting HRV. Additional recordings of HRV during the therapies and continues stress level questionnaires are recommended. In the light of these results, we also suggest synchronized multiple physiological recordings systems to be used in long-term studies. This first study showed that both therapies performed in water, in this population and setting was not effective in increasing HRV parameters. There is a substantial need for evidence-based research evaluating the specific effects of aquatic interventions on ANS activity in this population.

\section{Limitations}

The following limitations of this study need to be addressed. Measures of HRV by administering a 5-minute supine electrocardiogram is considered representative of 24-hour ambulatory recordings. Direct comparison with other studies is difficult, due to lack of standardized method for the frequency domain measures and therefore no universal unit. Both participants and assessors were not blinded. Possible influences of diet and energy intake were not examined in this study.

\section{Conclusion}

These findings suggest that multiple physiological recordings system should be a consideration in long-term HRV studies. In order to prescribe such therapies for obese population in warm water, longitudinal research with repetitive recordings to establish a specific therapy duration for obesity is required. Even though mechanistic and physiopathological studies strongly support aquatic interventions as promising approach, contrasting results in preliminary clinical trials exploring ANS modulation and aquatic interventions should be further investigated.

\section{References}

Adamson, P. B., Smith, A. L., Abraham, W. T., Kleckner, K. J., Stadler, R. W., Shih, A., \& Rhodes, M. M. (2004). Continuous autonomic assessment in patients with symptomatic heart failure. Circulation, 110(16), 2389-2394. https://doi.org/10.1161/01.CIR.0000139841.42454.78

Al Haddad, H., Laursen, P. B., Chollet, D., Lemaitre, F., Ahmaidi, S., \& Buchheit, M. (2010). Effect of cold or thermoneutral water immersion on post-exercise heart rate recovery and heart rate variability indices. Autonomic Neuroscience, 156(1), 111-116. https://doi.org/10.1016/j.autneu.2010.03.017

Bahman Bijari, B., Iranmanesh, S., Eshghi, F., \& Baneshi, M. R. (2012). Gentle human touch and yakson: the effect on preterm's behavioral reactions. ISRN nursing, 2012. https://doi.org/10.5402/2012/750363

Barbosa; Gustavo Augusto Seabra, et al. Effect of Watsu therapy on psychological aspects and quality of life of patients with temporomandibular disorder: case report. RGO-Revista Gaúcha de Odontologia, 2014, 62(3), 309-314.

Becker, B. E. (2009). Aquatic therapy: scientific foundations and clinical rehabilitation applications. $P M \& R, 1(9), 859-872$. https://doi.org/10.1016/j.pmrj.2009.05.017

Becker, B. E., Hildenbrand, K., Whitcomb, R. K., \& Sanders, J. P. (2009). Biophysiologic effects of warm water immersion. International Journal of Aquatic Research and Education, 3(1), 4. https://doi.org/10.25035/ijare.03.01.04

Björnsdotter, M., Morrison, I., \& Olausson, H. (2010). Feeling good: on the role of C fiber mediated touch in interoception. Experimental brain research, 207(3-4), 149-155. https://doi.org/10.1007/s00221-010-2408-y

Buchheit, M., \& Gindre, C. (2006). Cardiac parasympathetic regulation: respective associations with cardiorespiratory 
fitness and training load. American Journal of Physiology-Heart and Circulatory Physiology, 291(1), H451-H458. https://doi.org/10.1152/ajpheart.00008.2006

Canale, M. P., Manca di Villahermosa, S., Martino, G., Rovella, V., Noce, A., De Lorenzo, A., \& Di Daniele, N. (2013). http://doi.org/10.1155/2013/865965, Obesity-related metabolic syndrome: mechanisms of sympathetic overactivity. International journal of endocrinology, 2013. https://doi.org/10.1155/2013/865965

Chen, J. L., Chiu, H. W., Tseng, Y. J., \& Chu, W. C. (2006). Hyperthyroidism is characterized by both increased sympathetic and decreased vagal modulation of heart rate: evidence from spectral analysis of heart rate variability. Clinical endocrinology, 64(6), 611-616. https://doi.org/10.1111/j.1365-2265.2006.02514.x

Chon, S. C., Oh, D. W., \& Shim, J. H. (2009). Watsu approach for improving spasticity and ambulatory function in hemiparetic patients with stroke. Physiotherapy Research International, 14(2), 128-136. https://doi.org/10.1002/pri.421

Cider, Å., Sveälv, B. G., Täng, M. S., Schaufelberger, M., \& Andersson, B. (2006). Immersion in warm water induces improvement in cardiac function in patients with chronic heart failure. European journal of heart failure, 8(3), 308-313. https://doi.org/10.1016/j.ejheart.2005.08.001

de Oliveira Ottone, V., de Castro Magalhães, F., de Paula, F., Avelar, N. C. P., Aguiar, P. F., da Matta Sampaio, P. F., ... \& Nakamura, F. Y. (2014). The effect of different water immersion temperatures on post-exercise parasympathetic reactivation. PloS one, 9(12), e113730. https://doi.org/10.1371/journal.pone.0113730

Ditzen, B., Neumann, I. D., Bodenmann, G., von Dawans, B., Turner, R. A., Ehlert, U., \& Heinrichs, M. (2007). Effects of different kinds of couple interaction on cortisol and heart rate responses to stress in women. Psychoneuroendocrinology, 32(5), 565-574. https://doi.org/10.1016/j.psyneuen.2007.03.011

Dong, J. G. (2016). The role of heart rate variability in sports physiology. Experimental and Therapeutic Medicine, 11(5), 1531-1536. https://doi.org/10.3892/etm.2016.3104

Dull, H. (2004). Watsu: freeing the body in water. Trafford Publishing.

Eckel, R. H., York, D. A., Rössner, S., Hubbard, V., Caterson, I., Jeor, S. T. S., ... \& Blair, S. N. (2004). Prevention Conference VII. Circulation, 110(18), 2968-2975. https://doi.org/10.1161/01.CIR.0000140086.88453.9A

Faull, K. (2005). A pilot study of the comparative effectiveness of two water-based treatments for fibromyalgia syndrome: Watsu and Aix massage. Journal of Bodywork and Movement Therapies, 9(3), 202-210. https://doi.org/10.1016/j.jbmt.2004.12.001

Geytenbeek, J. (2002). Evidence for effective hydrotherapy. Physiotherapy, 88(9), 514-529. https://doi.org/10.1016/S0031-9406(05)60134-4

Goldstein, D. S., Bentho, O., Park, M. Y., \& Sharabi, Y. (2011). Low-frequency power of heart rate variability is not a measure of cardiac sympathetic tone but may be a measure of modulation of cardiac autonomic outflows by baroreflexes. Experimental physiology, 96(12), 1255-1261. https://doi.org/10.1113/expphysiol.2010.056259

Guarino, D., Nannipieri, M., Iervasi, G., Taddei, S., \& Bruno, R. M. (2017). The Role of the Autonomic Nervous System in the Pathophysiology of Obesity. Frontiers in physiology, 8, 665. https://doi.org/10.3389/fphys.2017.00665

Hadase, M., Azuma, A., Zen, K., Asada, S., Kawasaki, T., Kamitani, T., ... \& Matsubara, H. (2004). Very low frequency power of heart rate variability is a powerful predictor of clinical prognosis in patients with congestive heart failure. Circulation Journal, 68(4), 343-347. https://doi.org/10.1253/circj.68.343

Hall, J. E., Crook, E. D., Jones, D. W., Wofford, M. R., \& Dubbert, P. M. (2002). Mechanisms of obesity-associated cardiovascular and renal disease. The American journal of the Medical Sciences, 324(3), 127-137. https://doi.org/10.1097/00000441-200209000-00003

Hall, J. E., do Carmo, J. M., da Silva, A. A., Wang, Z., \& Hall, M. E. (2015). Obesity-induced hypertension: interaction of neurohumoral and renal mechanisms. Circulation Research, 116(6), 991. https://doi.org/10.1161/CIRCRESAHA.116.305697

Inoue, K; Udagawa, J; Wakasugi, N; Endo, K. (2013); The effect of longer cold water immersion time in the contrast bath on the skin temperature and parasympathetic activity Proceedings of The Physiological Society. Human Physiology Abstracts. 37th Congress of IUPS (Birmingham, UK) Proc 37th IUPS, PCD235

Kalil, G. Z., \& Haynes, W. G. (2012). Sympathetic nervous system in obesity-related hypertension: mechanisms and clinical implications. Hypertension Research, 35(1), 4-16. https://doi.org/10.1038/hr.2011.173

Kamath, C. (2015). A Novel Approach to Detect Congestive Heart Failure using Dispersion Entropy. J. Cardiovasc Res., 4, 5. https://dx. doi. org/10.4172/2324, 8602, 2 
Kamioka, H., Tsutani, K., Okuizumi, H., Mutoh, Y., Ohta, M., Handa, S., ... \& Honda, T. (2010). Effectiveness of aquatic exercise and balneotherapy: a summary of systematic reviews based on randomized controlled trials of water immersion therapies. Journal of Epidemiology, 20(1), 2-12. https://doi.org/10.2188/jea.JE20090030

Kelly, K. P., \& Kirschenbaum, D. S. (2011). Immersion treatment of childhood and adolescent obesity: the first review of a promising intervention. Obesity Reviews, 12(1), 37-49. https://doi.org/10.1111/j.1467-789X.2009.00710.x

Kenney, M. J., \& Ganta, C. K. (2014). Autonomic nervous system and immune system interactions. Comprehensive Physiology. https://doi.org/10.1002/cphy.c130051

Kesiktas, N., Paker, N., Erdogan, N., Gülsen, G., Biçki, D., \& Yilmaz, H. (2004). The use of hydrotherapy for the management of spasticity. Neurorehabilitation and Neural Repair, 18(4), 268-273. https://doi.org/10.1177/1545968304270002

Kjellgren, A., Sundequist, U., Norlander, T., \& Archer, T. (2001). Effects of flotation-REST on muscle tension pain. Pain Research and Management, 6(4), 181-189. https://doi.org/10.1155/2001/768501

Kluttig, A., Schumann, B., Swenne, C. A., Kors, J. A., Kuss, O., Schmidt, H., ... \& Greiser, K. H. (2010). Association of health behaviour with heart rate variability: a population-based study. BMC Cardiovascular Disorders, 10(1), 58. https://doi.org/10.1186/1471-2261-10-58

Lim, J. Y., Tchai, E., \& Jang, S. N. (2010). Effectiveness of aquatic exercise for obese patients with knee osteoarthritis: a randomized controlled trial. $P M \& R, 2(8), 723-731$. https://doi.org/10.1016/j.pmrj.2010.04.004

Maczkowiak, S., Hölter, G., \& Otten, H. (2007). WATSU-The effect of differently accentuated movement therapy interventions on clinically depressive patients. Bewegungstherapie und Gesundheitssport, 23(2), 58-64. https://doi.org/10.1055/s-2007-960607

McCraty, R., \& Shaffer, F. (2015). Heart rate variability: new perspectives on physiological mechanisms, assessment of self-regulatory capacity, and health risk. Global Advances in Health and Medicine, 4(1), 46-61. https://doi.org/10.7453/gahmj.2014.073

Metelka, R. (2014). Heart rate variability-current diagnosis of the cardiac autonomic neuropathy. A review. Biomedical Papers, 158(3), 327-338. https://doi.org/10.5507/bp.2014.025

Montagna, J. C., Santos, B. C., Battistuzzo, C. R., \& Loureiro, A. P. C. (2014). Effects of aquatic physiotherapy on the improvement of balance and corporal symmetry in stroke survivors. International journal of clinical and experimental medicine, 7(4), 1182.

Mooventhan, A., \& Nivethitha, L. (2014). Scientific evidence-based effects of hydrotherapy on various systems of the body. North American journal of medical sciences, 6(5), 199. https://doi.org/10.4103/1947-2714.132935

Mourot, L., Bouhaddi, M., Gandelin, E., Cappelle, S., Dumoulin, G., Wolf, J. P., ... \& Regnard, J. (2008). Cardiovascular autonomic control during short-term thermoneutral and cool head-out immersion. Aviation, space, and environmental medicine, 79(1), 14-20. https://doi.org/10.3357/ASEM.2147.2008

Moyer, C. A., Rounds, J., \& Hannum, J. W. (2004). A meta-analysis of massage therapy research.

Nagasawa, Y., Komori, S., Sato, M., Tsuboi, Y., Umetani, K., Watanabe, Y., \& Tamura, K. (2001). Effects of hot bath immersion on autonomic activity and hemodynamics. Japanese Circulation Journal, 65(7), 587-592. https://doi.org/10.1253/jcj.65.587

Norris, P. R., Morris, J. A., Ozdas, A., Grogan, E. L., \& Williams, A. E. (2005). Heart rate variability predicts trauma patient outcome as early as $12 \mathrm{~h}$ : implications for military and civilian triage. Journal of Surgical Research, 129(1), 122-128. https://doi.org/10.1016/j.jss.2005.04.024

Poirier, P., \& Eckel, R. H. (2002). Obesity and cardiovascular disease. Current atherosclerosis reports, 4(6), 448-453. https://doi.org/10.1007/s11883-002-0049-8

Poirier, P., Martin, J., Marceau, P., Biron, S., \& Marceau, S. (2004). Impact of bariatric surgery on cardiac structure, function and clinical manifestations in morbid obesity. Expert Review of Cardiovascular Therapy, 2(2), 193-201. https://doi.org/10.1586/14779072.2.2.193

Rennie, K. L., Hemingway, H., Kumari, M., Brunner, E., Malik, M., \& Marmot, M. (2003). Effects of moderate and vigorous physical activity on heart rate variability in a British study of civil servants. American Journal of Epidemiology, 158(2), 135-143. https://doi.org/10.1093/aje/kwg120

Reyes del Paso, G. A., Langewitz, W., Mulder, L. J., Roon, A., \& Duschek, S. (2013). The utility of low frequency heart rate variability as an index of sympathetic cardiac tone: a review with emphasis on a reanalysis of previous studies. Psychophysiology, 50(5), 477-487. https://doi.org/10.1111/psyp.12027 
Rossi, R. C., Vanderlei, L. C. M., Gonçalves, A. C. C. R., Vanderlei, F. M., Bernardo, A. F. B., Yamada, K. M. H., ... \& de Abreu, L. C. (2015). Impact of obesity on autonomic modulation, heart rate and blood pressure in obese young people. Autonomic Neuroscience, 193, 138-141. https://doi.org/10.1016/j.autneu.2015.07.424

Schipke, J. D., \& Pelzer, M. (2001). Effect of immersion, submersion, and scuba diving on heart rate variability. British Journal of Sports Medicine, 35(3), 174-180. https://doi.org/10.1136/bjsm.35.3.174

Schitter, A. M., Nedeljkovic, M., Baur, H., Fleckenstein, J., \& Raio, L. (2015). Effects of passive hydrotherapy WATSU (WaterShiatsu) in the third trimester of pregnancy: results of a controlled pilot study. Evidence-Based Complementary and Alternative Medicine, 2015. https://doi.org/10.1155/2015/437650

Schoedinger, P., \& potential risks when utilizing Watsu, D. (2011). Watsu in aquatic rehabilitation. Comprehen-sive Aquatic Therapy, AJ Cole and BE Becker, Eds.

Shaffer, F., \& Ginsberg, J. P. (2017). An overview of heart rate variability metrics and norms. Frontiers in public health, 5, 258. https://doi.org/10.3389/fpubh.2017.00258

Sherman, K. J., Ludman, E. J., Cook, A. J., Hawkes, R. J., Roy-Byrne, P. P., Bentley, S., ... \& Cherkin, D. C. (2010). Effectiveness of therapeutic massage for generalized anxiety disorder: a randomized controlled trial. Depression and anxiety, 27(5), 441-450. https://doi.org/10.1002/da.20671

Smith, M. M., \& Minson, C. T. (2012). Obesity and adipokines: effects on sympathetic overactivity. The Journal of Physiology, 590(8), 1787-1801. https://doi.org/10.1113/jphysiol.2011.221036

Soni, K, Shetty, P,. Sujatha K.J.A (2016), Comparative Study on Effects of Neutral And Cold Underwater Massage on the Physiological Parameters of Healthy Individuals IOSR Journal of Dental and Medical Sciences e-ISSN: 2279-0853, p-ISSN: 2279-0861.Volume 15, Issue 9 Ver. X, PP 32-36

Stan, E. A. (2013). Effects of water watsu applications for people with impairment. Sport \& Society/Sport si Societate, 13(1).

Sveälv, B. G., Cider, Å., Täng, M. S., Angwald, E., Kardassis, D., \& Andersson, B. (2009). Benefit of warm water immersion on biventricular function in patients with chronic heart failure. Cardiovascular Ultrasound, 7(1), 33. https://doi.org/10.1186/1476-7120-7-33

Task Force of the European Society of Cardiology the North American Society of Pacing Electrophysiology. (1996) Heart Rate Variability: Standards of Measurement, Physiological Interpretation, and Clinical Use Circulation.1996; 93: 1043-1065

Thayer, J. F., Hansen, A. L., Saus-Rose, E., \& Johnsen, B. H. (2009). Heart rate variability, prefrontal neural function, and cognitive performance: the neurovisceral integration perspective on self-regulation, adaptation, and health. Annals of Behavioral Medicine, 37(2), 141-153. https://doi.org/10.1007/s12160-009-9101-z

Torres-Ronda, L., \& Schelling i del Alcázar, X. (2014). The properties of water and their applications for training. Journal of Human Kinetics, 44(1), 237-248. https://doi.org/10.2478/hukin-2014-0129

Tufekcioglu, E. and Hassanain M.B.M., (2016) Acute effect of single Watsu therapy on the flexibility of young male swimmers. European College of Sports Science Congress Vienna,

Tufekcioglu, E., \& Çotuk, H. B. (2009). Suda ve karadaki farkli beden konumlarinda kalp hızı değişkenliğinin karşılaştırılması. Beden Egitimi ve Spor Bilimleri Dergisi, 3(3).

Tufekcioglu, E., Cotuk B., Erzeybek, M., Kaya F., Pinar, S.,(2010). Effect of Watsu Therapy on Psychological State. National Thermal medicine and balneology congress. Cesme.

Umphred, D. A. (1995). Classification of treatment techniques based on primary input systems. Neurological rehabilitation, 3rd edn. Mosby, St. Louis, 118-178.

Windham, B. G., Fumagalli, S., Ble, A., Sollers, J. J., Thayer, J. F., Najjar, S. S., ... \& Ferrucci, L. (2012). The relationship between heart rate variability and adiposity differs for central and overall adiposity. Journal of Obesity, 2012. https://doi.org/10.1155/2012/149516

Zamunér, A. R., Andrade, C. P., Forti, M., Marchi, A., Milan, J., Avila, M. A., ... \& Silva, E. (2015). Effects of a hydrotherapy programme on symbolic and complexity dynamics of heart rate variability and aerobic capacity in fibromyalgia patients. Clin Exp Rheumatol, 33(88), S73-S81.

Ziegler, D., Zentai, C., Perz, S., Rathmann, W., Haastert, B., Meisinger, C., ... \& KORA Study Group. (2006). Selective contribution of diabetes and other cardiovascular risk factors to cardiac autonomic dysfunction in the general population. Experimental and Clinical Endocrinology \& Diabetes, 114(04), 153-159.

https://doi.org/10.1055/s-2006-924083 
Zimmermann, F. (2015). Mindfulness-Based Practices as a Resource for Health and Well-Being. Medical Acupuncture, 27(5), 349-359. https://doi.org/10.1089/acu.2014.1080

\section{Copyrights}

Copyright for this article is retained by the author(s), with first publication rights granted to the journal.

This is an open-access article distributed under the terms and conditions of the Creative Commons Attribution license which permits unrestricted use, distribution, and reproduction in any medium, provided the original work is properly cited. 